\title{
3 Research Square

\section{Real-World Comparison of CT-P6 and Reference Trastuzumab for the Treatment of HER2-Positive Early-Stage and Metastatic Breast Cancer}

\section{Soong June Bae}

Gangnam Severance Hospital

Jee Hung Kim

Gangnam Severance Hospital

Sung Gwe Ahn

Gangnam Severance Hospital

Hei-Cheul Jeung

Gangnam Severance Hospital

Joohyuk Sohn

Yonsei Cancer Center

Gun Min Kim

Yonsei Cancer Center

Min Hwan Kim

Yonsei Cancer Center

Seung II Kim

Yonsei Cancer Center

Seho Park

Yonsei Cancer Center

Hyung Seok Park

Yonsei Cancer Center

Jee Ye Kim

Yonsei Cancer Center

Joon Jeong ( $\sim$ gsjjoon@yuhs.ac)

Gangnam Severance Hospital, Yonsei University College of Medicine https://orcid.org/0000-00030397-0005

Research article

Keywords: CT-P6, trastuzumab, biosimilar, HER2-positive breast cancer, neoadjuvant, metastatic

Posted Date: March 3rd, 2021 
DOl: https://doi.org/10.21203/rs.3.rs-268509/v1

License: (c) (1) This work is licensed under a Creative Commons Attribution 4.0 International License. Read Full License 


\section{Abstract}

Background: Here, we present the first real-world comparison of CT-P6 versus reference trastuzumab (RTZ) for the neoadjuvant treatment of patients with HER2-positive EBC, and for the palliative first-line treatment of patients with HER2-positive metastatic breast cancer (MBC).

Methods: We retrospectively identified patients with HER2-positive EBC who had received neoadjuvant treatment with RTZ or CT-P6, plus pertuzumab, carboplatin, and docetaxel, followed by surgery, alongside patients with newly diagnosed HER2-positive MBC who had received palliative treatment with RTZ or CTP6, plus pertuzumab and docetaxel. The primary endpoints were pathologic complete response ( $p C R)$ in the EBC cohort, and progression-free survival (PFS) in the MBC cohort.

Results: A similar percentage of patients with EBC achieved a pCR with CT-P6 (74.4\% [93/125]) and RTZ (69.8\% [90/129]) ( $p=0.411)$. For patients with MBC, median PFS did not differ significantly between the two groups (CT-P6: 13.9 months [95\% confidence intervals (Cls) not available]; RTZ: 18.4 months [95\% Cls 12.5-24.3]; $p=0.653)$. The cardiac safety profiles of CT-P6 and RTZ were similar.

Conclusions: These real-world data suggest that CT-P6 has similar effectiveness and cardiac safety to RTZ in patients with HER2-positive EBC and MBC, when administered as part of dual HER2-targeted therapy with pertuzumab plus chemotherapy in the neoadjuvant or palliative setting.

\section{Background}

Breast cancer is a heterogeneous disease that has multiple subtypes. Approximately $25 \%$ of breast cancers amplify the human epidermal growth factor receptor 2 (HER2) oncogene, resulting in a more aggressive phenotype with poorer prognosis.[1] The development of trastuzumab, a humanized monoclonal antibody that binds to the HER2 extracellular domain, has transformed the treatment of HER2-positive breast cancers.[2] In patients with operable or locally advanced HER2-positive disease, the addition of trastuzumab to chemotherapy improves clinical responses and event-free survival in the neoadjuvant setting[2-4] and long-term disease-free survival in the adjuvant setting.[5] Trastuzumab is also effective in metastatic HER2-positive breast cancer, with the addition of trastuzumab to standard chemotherapy shown to extend time to disease progression, as well as overall survival (OS).[6, 7]

However, the development of novel biologic agents is expensive, which can translate into high drug costs. [8] Despite their efficacy, the costs associated with these drugs can pose a burden on healthcare systems and create barriers to access. This difficulty can be mitigated in part through the use of biosimilars.[8] A biosimilar is a drug that is highly similar to an existing drug - the originator or reference product - and which shows no clinically meaningful differences from the reference product in purity, safety, and efficacy.[8] Biosimilars are in general more affordable than their reference products, and their availability has the potential to improve patient access to safe and effective treatments. 
Several biosimilars of trastuzumab have been developed, including CT-P6 (Herzuma ${ }^{\circledR}$; Celltrion Inc., Incheon, Republic of Korea),[9, 10] SB3 (Ontruzant®; Samsung Bioepis Co., Ltd, Incheon, Republic of Korea),[11] ABP 980 (Kanjinti®; Amgen, Thousand Oaks, CA, USA)[12] and PF-05280014 (Trazimera ${ }^{\circledR}$; Pfizer, New York City, NY, USA).[13] CT-P6 binds with high affinity and specificity to the same HER2 epitope as the reference product, Herceptin ${ }^{\circledR}$ (Genentech, San Francisco, CA, USA). [9, 10] In 2018, CT-P6 was approved by the US Food and Drug Administration and the European Medicines Agency for the treatment of HER2-positive early-stage breast cancer (EBC) and metastatic breast cancer (MBC).[14, 15] Approval was based in part on a phase III clinical trial in patients with operable HER2-positive EBC, which showed comparable safety and equivalent efficacy of CT-P6 and reference trastuzumab (RTZ) when administered in combination with docetaxel and fluorouracil, epirubicin and cyclophosphamide (FEC).[16, 17] Data from a 3-year follow-up study have subsequently confirmed that disease-free survival and OS are similar in patients treated with CT-P6 and RTZ.[18]

Real-world studies are an important supplement to clinical trials, by revealing the long-term safety and effectiveness of drugs in broader patient populations, as well as in other settings and in combination with other treatments. For example, RTZ is now increasingly used in combination with pertuzumab (Perjeta®; Genentech) as part of dual HER2-targeted therapy. Pertuzumab is a monoclonal antibody that targets a different region of the HER2 receptor to trastuzumab,[19] and thus has a complementary mode of action. Dual HER2-targeting with trastuzumab and pertuzumab, plus chemotherapy, has been shown to improve clinical responses compared to trastuzumab plus chemotherapy alone in HER2-positive EBC[20, 21] and MBC.[22]

Here we present the results of the first real-world comparison of the effectiveness of CT-P6 and RTZ when administered with pertuzumab plus chemotherapy in the neoadjuvant setting to patients with HER2positive EBC, and as palliative first-line treatment to patients with HER2-positive MBC. As cardiotoxicity is a potentially serious adverse effect associated with the use of RTZ,[6] we also assessed the cardiac safety of CT-P6 and the reference product.

\section{Methods}

\section{Patients}

We retrospectively reviewed the medical records in the $\mathrm{EBC}$ and $\mathrm{MBC}$ cohort from Severance Breast Cancer Registry at the Yonsei Cancer Center and Gangnam Severance Hospital in Seoul, Republic of Korea (Additional file 1).

We identified patients with HER2-positive EBC who had undergone neoadjuvant chemotherapy between April 2015 and October 2019, as well as patients with newly diagnosed de novo or recurrent HER2positive MBC who had undergone palliative chemotherapy between May 2014 and December 2019. For both the EBC and MBC cohorts, eligible patients were women aged $>19$ years with a histologically confirmed diagnosis of HER2-positive breast cancer. 
EBC was defined as clinical stage II-III, classified according to the American Joint Committee on Cancer Breast Cancer Staging seventh edition. In the EBC cohort, eligible women had received neoadjuvant treatment with docetaxel $(T)$ - carboplatin (C)-RTZ or CT-P6 (H) - pertuzumab (P) (TCHP) chemotherapy followed by surgery, and had a primary tumor $>2 \mathrm{~cm}$ in diameter or clinically metastatic axillary lymph node-positive cancer. Patients were excluded from the EBC cohort if they had bilateral breast cancer or a history of ductal carcinoma in situ (DCIS) or invasive breast cancer.

In the MBC cohort, eligible women had received palliative first-line treatment with docetaxel $(T)-R T Z$ or CT-P6 (H)-pertuzumab (P) (THP) chemotherapy.

Patients with MBC were still eligible if they had received chemotherapy with or without trastuzumab in the neoadjuvant or adjuvant setting, provided there was a minimum 12-month interval between completion of all therapy and the diagnosis of metastatic disease.

Patients were excluded from the MBC cohort if they had received RTZ and docetaxel as first-line palliative treatment, or if they had switched from RTZ to CT-P6 during treatment.

\section{Procedures}

In the EBC cohort, neoadjuvant TCHP treatment was administered via intravenous infusion every 3 weeks, for a total of six cycles, in accordance with procedures in the TRYPHAENA trial.

[23] Study drugs were given consecutively on the same day in the following sequence: CT-P6 or RTZ, followed by pertuzumab, carboplatin, and docetaxel. CT-P6 or RTZ were administered at a loading dose of $8 \mathrm{mg} / \mathrm{kg}$ on day 1 of cycle 1 , and at a maintenance dose of $6 \mathrm{mg} / \mathrm{kg}$ on day 1 of cycles 2-6. Pertuzumab was administered at a loading dose of $840 \mathrm{mg}$, then a maintenance dose of $420 \mathrm{mg}$ in subsequent cycles. Carboplatin was administered at area under the curve 5 or 6 and docetaxel at $75 \mathrm{mg} / \mathrm{m}^{2}$, in all cycles.

In the MBC cohort, palliative first-line treatment with THP was administered via intravenous infusion every 3 weeks, in accordance with procedures in the CLEOPATRA trial.

[24] Pertuzumab was given on day 1 of each cycle, at a loading dose of $840 \mathrm{mg}$ in cycle 1, decreasing to a maintenance dose of $420 \mathrm{mg}$ in subsequent cycles. RTZ or CT-P6 were administered on day 1 of each cycle at a loading dose of $8 \mathrm{mg} / \mathrm{kg}$, and then a maintenance dose of $6 \mathrm{mg} / \mathrm{kg}$ in subsequent cycles. Docetaxel was given on day 1 of the first cycle at $75 \mathrm{mg} / \mathrm{m}^{2}$. Pertuzumab and RTZ or CT-P6 were given until disease progression or unmanageable toxic effects, and docetaxel was given for at least six cycles. If chemotherapy was discontinued due to toxic effects, pertuzumab and RTZ or CT-P6 were continued until disease progression, or occurrence of unacceptable adverse events.

\section{Study objectives and assessments}

The study objective was to compare the effectiveness and cardiac safety of CT-P6 and RTZ in patients with HER2-positive EBC and MBC, when administered as part of a chemotherapy regimen containing pertuzumab in the neoadjuvant or first-line palliative setting, respectively. 
In the EBC cohort, tumor assessments were performed at baseline and after completion of cycle 6 of the neoadjuvant regimen prior to surgery, by ultrasonography or magnetic resonance imaging and clinical breast examination. In the MBC cohort, tumor assessment was performed every 9 weeks based on the Response Evaluation Criteria in Solid Tumors version 1.1 (RECIST 1.1). Left ventricular ejection fraction (LVEF) was measured either by echocardiography or a multiple-gated acquisition scan every 3 months, with the same method always used for individual patients.

\section{Outcome measures}

The primary endpoints were pathologic complete response (pCR) in the EBC cohort, and progression-free survival (PFS) in the MBC cohort. PCR was assessed locally at the time of surgery and was defined as the absence of invasive tumor cells during a microscopic assessment of the primary tumor (ypT0/is) and axilla (ypNO). Median PFS was defined as time from the date of first-line palliative systemic treatment to the first documented disease progression or death from any cause.

The secondary endpoints in the MBC cohort were median overall survival (mOS), overall response rate (ORR) and disease control rate (DCR). mOS was the time from the date of first-line palliative systemic treatment to death from any cause. ORR was defined as the proportion of patients who achieved a complete response (CR) or confirmed partial response (CPR) per RECIST 1.1. DCR was defined as the proportion of patients who achieved a CR, CPR, or stable disease (SD) per RECIST 1.1.

Cardiac safety was a secondary endpoint in both the EBC and MBC cohorts. An adverse event related to cardiac safety was defined as a decline in investigator-assessed LVEF of $\geq 10$ percentage points from baseline at any time, or an LVEF of $<50 \%$ at any time.

\section{Statistical analysis}

Standard descriptive and analytic methods were used to describe the patient population and their baseline characteristics. PCR rate was compared between treatment groups using the chi-square test or Fisher's exact test. To reduce baseline confounders between the CT-P6 group and RTZ group, one-to-one propensity score matching (PSM) was performed using the nearest-neighbor matching method. Variables entered into the PSM included age, histologic type, histologic grade, estrogen receptor, progesterone receptor, subgroup, Ki67, clinical tumor stage, and clinical node stage. PFS and OS were evaluated using unadjusted log-rank tests and Cox proportional-hazards models. Differences in ORR or DCR between the treatment groups were evaluated using an adjusted Fisher's exact test. The 95\% confidence intervals (Cls) for the ORRs were calculated using an asymptotic normal approximation. A two-tailed $P$-value $<0.05$ was considered statistically significant. The statistical analysis was performed using SPSS version 23 software (SPSS Inc., Chicago, IL, USA).

\section{Results}

\section{EBC cohort}


A total of 258 women with HER2-positive EBC were identified who underwent neoadjuvant treatment with TCHP, followed by surgery, between April 2015 and October 2019 (Additional file 1).

Four patients were excluded owing to the presence of bilateral breast lesions $(n=3)$ or a history of DCIS/invasive breast cancer $(n=1)$. The remaining 254 women were analyzed in this study.

Baseline characteristics were similar between patients who received CT-P6 $(n=125,49.2 \%)$ and those receiving RTZ ( $n=129,50.8 \%$ ) (Table 1 ). The median (range) ages of patients in the CT-P6 and RTZ groups were 50 (27-71) years and 49 (22-75) years, respectively. Approximately three-quarters of patients in both groups had histologic grade 1 or 2 tumors. A similar percentage of patients in each treatment group had hormone receptor (HR)-positive breast cancer (34.9\% in the RTZ group and $36.0 \%$ in the CT-P6 group). A greater proportion of patients in the CT-P6 treatment group had a Ki67 index $\geq 14$ (CTP6: 87.5\% [84/125]; RTZ: 70.5\% [55/129]; $P=0.005)$. After adjusting for propensity scores, all variables were well balanced between the CT-P6 group and RTZ group (Additional file 2). 
Table 1

Baseline characteristics of patients with HER2-positive early-stage and metastatic breast cancer Early-stage breast cancer

Metastatic breast cancer

\begin{tabular}{|c|c|c|c|c|c|c|c|c|}
\hline & $\begin{array}{l}\text { RTZ } \\
(n= \\
129)\end{array}$ & $\begin{array}{l}\text { CT-P6 (n } \\
=125)\end{array}$ & $\begin{array}{l}\text { Total } \\
(\mathrm{N}= \\
254)\end{array}$ & $\begin{array}{l}\mathrm{P}- \\
\text { value }\end{array}$ & $\begin{array}{l}\text { RTZ } \\
(n= \\
65)\end{array}$ & $\begin{array}{l}\text { CT-P6 } \\
(n= \\
38)\end{array}$ & $\begin{array}{l}\text { Total } \\
(\mathrm{N}= \\
103)\end{array}$ & $\begin{array}{l}\mathrm{P} \text { - } \\
\text { value }\end{array}$ \\
\hline $\begin{array}{l}\text { Age, median } \\
\text { (range) }\end{array}$ & $\begin{array}{l}49 \\
(22- \\
75)\end{array}$ & $\begin{array}{l}50(27- \\
71)\end{array}$ & $\begin{array}{l}49 \\
(22- \\
75)\end{array}$ & 0.447 & $\begin{array}{l}54 \\
(31- \\
76)\end{array}$ & $\begin{array}{l}55 \\
(28- \\
79)\end{array}$ & $\begin{array}{l}54 \\
(28- \\
79)\end{array}$ & 0.695 \\
\hline Stage IV, $n(\%)$ & & & & & & & & 0.333 \\
\hline De novo & - & - & - & & $\begin{array}{l}47 \\
(72.3)\end{array}$ & $\begin{array}{l}24 \\
(63.2)\end{array}$ & $\begin{array}{l}71 \\
(68.9)\end{array}$ & \\
\hline Recurrent & - & - & - & & $\begin{array}{l}18 \\
(27.7)\end{array}$ & $\begin{array}{l}14 \\
(36.8)\end{array}$ & $\begin{array}{l}32 \\
(31.1)\end{array}$ & \\
\hline Histology, $n(\%)$ & & & & $\begin{array}{l}< \\
0.001\end{array}$ & & & & 0.117 \\
\hline IDC & $\begin{array}{l}113 \\
(87.6)\end{array}$ & $\begin{array}{l}124 \\
(99.2)\end{array}$ & $\begin{array}{l}237 \\
(93.3)\end{array}$ & & $\begin{array}{l}60 \\
(93.8)\end{array}$ & $\begin{array}{l}32 \\
(86.5)\end{array}$ & $\begin{array}{l}92 \\
(91.1)\end{array}$ & \\
\hline ILC & $\begin{array}{l}11 \\
(8.5)\end{array}$ & $1(0.8)$ & $\begin{array}{l}12 \\
(4.7)\end{array}$ & & $1(1.6)$ & $0(0)$ & $1(1.0)$ & \\
\hline Other ${ }^{a}$ & $5(3.9)$ & 0 & $5(2.0)$ & & $3(4.7)$ & $\begin{array}{l}5 \\
(13.5)\end{array}$ & $8(7.9)$ & \\
\hline $\mathrm{ER}, n(\%)$ & & & & 0.752 & & & & 0.804 \\
\hline Positive & $\begin{array}{l}44 \\
(34.1)\end{array}$ & $45(36.0)$ & $\begin{array}{l}89 \\
(35.0)\end{array}$ & & $\begin{array}{l}29 \\
(44.6)\end{array}$ & $\begin{array}{l}16 \\
(42.1)\end{array}$ & $\begin{array}{l}45 \\
(43.7)\end{array}$ & \\
\hline Negative & $\begin{array}{l}85 \\
(65.9)\end{array}$ & $80(64.0)$ & $\begin{array}{l}165 \\
(65.0)\end{array}$ & & $\begin{array}{l}36 \\
(55.4)\end{array}$ & $\begin{array}{l}22 \\
(57.9)\end{array}$ & $\begin{array}{l}58 \\
(56.3)\end{array}$ & \\
\hline $\mathrm{PR}, n(\%)$ & & & & 0.992 & & & & 0.812 \\
\hline Positive & $\begin{array}{l}30 \\
(23.3)\end{array}$ & $29(23.2)$ & $\begin{array}{l}59 \\
(23.2)\end{array}$ & & $\begin{array}{l}15 \\
(23.1)\end{array}$ & $\begin{array}{l}8 \\
(21.1)\end{array}$ & $\begin{array}{l}23 \\
(22.3)\end{array}$ & \\
\hline Negative & $\begin{array}{l}99 \\
(76.7)\end{array}$ & $96(76.8)$ & $\begin{array}{l}195 \\
(76.8)\end{array}$ & & $\begin{array}{l}50 \\
(76.9)\end{array}$ & $\begin{array}{l}30 \\
(78.9)\end{array}$ & $\begin{array}{l}80 \\
(77.7)\end{array}$ & \\
\hline Subgroup, $n(\%)$ & & & & 0.852 & & & & 0.804 \\
\hline HR+/HER2+ & $\begin{array}{l}45 \\
(34.9)\end{array}$ & $45(36.0)$ & $\begin{array}{l}90 \\
(35.4)\end{array}$ & & $\begin{array}{l}29 \\
(44.6)\end{array}$ & $\begin{array}{l}16 \\
(42.1)\end{array}$ & $\begin{array}{l}45 \\
(43.7)\end{array}$ & \\
\hline HR-/HER2+ & $\begin{array}{l}84 \\
(65.1)\end{array}$ & $80(64.0)$ & $\begin{array}{l}164 \\
(64.6)\end{array}$ & & $\begin{array}{l}36 \\
(55.4)\end{array}$ & $\begin{array}{l}22 \\
(57.9)\end{array}$ & $\begin{array}{l}58 \\
(56.3)\end{array}$ & \\
\hline
\end{tabular}




\begin{tabular}{|c|c|c|c|c|c|c|c|c|}
\hline \multirow[b]{2}{*}{$\mathrm{HG},{ }^{\mathrm{b}} n(\%)$} & \multicolumn{4}{|c|}{ Early-stage breast cancer } & \multicolumn{4}{|c|}{ Metastatic breast cancer } \\
\hline & & & & 0.960 & & & & 0.135 \\
\hline 1 or 2 & $\begin{array}{l}68 \\
(73.1)\end{array}$ & $83(72.8)$ & $\begin{array}{l}151 \\
(72.9)\end{array}$ & & $\begin{array}{l}4 \\
(36.4)\end{array}$ & $\begin{array}{l}7 \\
(70.0)\end{array}$ & $\begin{array}{l}11 \\
(52.4)\end{array}$ & \\
\hline 3 & $\begin{array}{l}25 \\
(26.9)\end{array}$ & $31(27.2)$ & $\begin{array}{l}56 \\
(27.1)\end{array}$ & & $\begin{array}{l}7 \\
(63.6)\end{array}$ & $\begin{array}{l}3 \\
(30.0)\end{array}$ & $\begin{array}{l}10 \\
(47.6)\end{array}$ & \\
\hline $\mathrm{Ki} 67,{ }^{\mathrm{b}} n(\%)$ & & & & 0.005 & & & & 0.647 \\
\hline$<14$ & $\begin{array}{l}23 \\
(29.5)\end{array}$ & $12(12.5)$ & $\begin{array}{l}35 \\
(20.1)\end{array}$ & & $\begin{array}{l}3 \\
(11.1)\end{array}$ & $\begin{array}{l}3 \\
(11.5)\end{array}$ & $\begin{array}{l}6 \\
(11.3)\end{array}$ & \\
\hline$\geq 14$ & $\begin{array}{l}55 \\
(70.5)\end{array}$ & $84(87.5)$ & $\begin{array}{l}139 \\
(79.9)\end{array}$ & & $\begin{array}{l}24 \\
(88.9)\end{array}$ & $\begin{array}{l}23 \\
(88.5)\end{array}$ & $\begin{array}{l}47 \\
(88.7)\end{array}$ & \\
\hline $\mathrm{cT}^{\mathrm{c}}{ }^{n}(\%)$ & & & & 0.139 & & & & \\
\hline 1 or 2 & $\begin{array}{l}85 \\
(65.9)\end{array}$ & $93(74.4)$ & $\begin{array}{l}178 \\
(70.1)\end{array}$ & & - & - & - & \\
\hline$\geq 3$ & $\begin{array}{l}44 \\
(34.1)\end{array}$ & $32(25.6)$ & $\begin{array}{l}76 \\
(29.9)\end{array}$ & & - & - & - & \\
\hline $\mathrm{cN},{ }^{\mathrm{d}} n(\%)$ & & & & 0.072 & & & & \\
\hline Negative & $\begin{array}{l}16 \\
(12.4)\end{array}$ & $26(20.8)$ & $\begin{array}{l}42 \\
(16.5)\end{array}$ & & - & - & - & \\
\hline Positive & $\begin{array}{l}113 \\
(87.6)\end{array}$ & $99(79.2)$ & $\begin{array}{l}212 \\
(83.5)\end{array}$ & & - & - & - & \\
\hline \multicolumn{9}{|l|}{$\begin{array}{l}\text { Metastasis site, } \\
n(\%)\end{array}$} \\
\hline Distant LN & - & - & - & & $\begin{array}{l}39 \\
(60.0)\end{array}$ & $\begin{array}{l}19 \\
(50.0)\end{array}$ & $\begin{array}{l}58 \\
(56.3)\end{array}$ & 0.323 \\
\hline Bone & - & - & - & & $\begin{array}{l}33 \\
(50.8)\end{array}$ & $\begin{array}{l}16 \\
(42.1)\end{array}$ & $\begin{array}{l}48 \\
(47.6)\end{array}$ & 0.396 \\
\hline Lung & - & - & - & & $\begin{array}{l}21 \\
(32.3)\end{array}$ & $\begin{array}{l}14 \\
(36.8)\end{array}$ & $\begin{array}{l}35 \\
(34.0)\end{array}$ & 0.639 \\
\hline Liver & - & - & - & & $\begin{array}{l}18 \\
(29.2)\end{array}$ & $\begin{array}{l}14 \\
(36.8)\end{array}$ & $\begin{array}{l}33 \\
(32.0)\end{array}$ & 0.424 \\
\hline Breast skin & - & - & - & & $4(6.2)$ & $\begin{array}{l}6 \\
(15.8)\end{array}$ & $\begin{array}{l}10 \\
(9.7)\end{array}$ & 0.107 \\
\hline Brain & - & - & - & & $4(6.2)$ & $3(7.9)$ & $7(6.8)$ & 0.513 \\
\hline Pleural & - & - & - & & $3(4.6)$ & $\begin{array}{l}4 \\
(10.5)\end{array}$ & $7(6.8)$ & 0.225 \\
\hline
\end{tabular}




\section{Early-stage breast cancer}

Other ${ }^{\mathrm{e}}$

$-\quad-$

Metastatic breast cancer

$2(5.3)$

11

$(10.7)$

0.151

a Other histology included micropapillary, apocrine, mucinous, and metaplastic carcinoma.

b Values were missing for some patients across groups.

${ }^{\mathrm{c}} \mathrm{cT}$ was based on tumor size measured by pre-treatment breast magnetic resonance imaging.

${ }^{\mathrm{d}} \mathrm{CN}$ positive was defined as axillary lymph node metastasis proven by fine-needle aspiration biopsy (FNAB), or suspicious axillary lymph node metastasis in the imaging study among patients who did not receive FNAB.

e Other metastasis sites included adrenal gland, peritoneum, and soft tissue mass.

cN, clinical node stage; cT, clinical tumor stage; ER, estrogen receptor; HER2, human epidermal growth factor receptor 2; HG, histologic grade; HR, hormone receptor; IDC, invasive ductal carcinoma; ILC, invasive lobular carcinoma; LN, lymph node; PR, progesterone receptor; RTZ, reference trastuzumab.

\section{Effectiveness}

The percentage of patients who achieved a pCR did not differ between the two treatment groups (Fig. 1A). Overall, 74.4\% (93/125) achieved a pCR with CT-P6 versus $69.8 \%(90 / 129)$ with RTZ $(P=0.411)$. Among patients with HR-positive breast cancer, $57.8 \%(26 / 45)$ of patients who received CT-P6 achieved a pCR, compared with $44.4 \%(20 / 45)$ of patients who received RTZ $(P=0.206)$. Among patients with HRnegative breast cancer, $83.8 \%(67 / 80)$ of patients who received CT-P6 achieved a pCR, as did $83.3 \%$ (70/84) of patients who received RTZ $(P=0.943)$. Similarly, there was no difference in pCR between the two groups in the PSM cohort, regardless of subtype (Fig. 1B).

Cardiac safety

The rate of LVEF did not differ between the two treatment groups before neoadjuvant treatment (mean [95\% CI] LVEF: CT-P6: 68.1\% [67.0-69.1] versus RTZ: 68.1\% [67.1-69.1]; $P=0.983$ ) or after neoadjuvant chemotherapy (CT-P6: 65.9\% [65.0-66.8] versus RTZ: 66.5\% [65.3-67.7]; $P=0.424$ ) (Additional file 3). After neoadjuvant chemotherapy, 23/104 (22.1\%) patients showed a decrease in LVEF of $\geq 10$ percentage points from baseline in the CT-P6 group, compared with 13/90 (14.4\%) patients in the RTZ group $(P=0.171)$. No patients in either treatment group showed a decrease in LVEF to $<50 \%$ at any point.

\section{MBC cohort}

Study population

A total of 138 women with recurrent or de novo stage IV HER2-positive MBC were identified who received palliative first-line treatment with THP between May 2014 and December 2019. Eighteen patients were excluded because they received either palliative RTZ monotherapy or palliative RTZ plus docetaxel. A 
further 17 patients were excluded because they were switched from RTZ to CT-P6. The remaining 103 women were analyzed in this study.

Baseline characteristics were similar for patients who received CT-P6 $(n=38,36.9 \%)$ and RTZ $(n=65$, $63.1 \%$ ) (Table 1). The median (range) age of patients in the CT-P6 group was 55 (28-79) years, compared with 54 (31-76) years in the RTZ group. The percentage of patients with de novo stage IV MBC was $63.2 \%(24 / 38)$ in the CT-P6 group and $72.3 \%(47 / 65)$ among those who received RTZ. A similar percentage of patients in each treatment group had HR-positive breast cancer (42.1\% in the CT-P6 group and $44.6 \%$ in the RTZ group). Among the patients for whom the histologic grade of tumors was known, $30.0 \%(3 / 10)$ in the CT-P6 group had grade 3 tumors, compared with $63.6 \%(7 / 11)$ in the RTZ group ( $P=$ 0.135). Almost $90 \%$ of patients in each treatment group had a Ki67 index $\geq 14$.

In both groups, the most common sites of metastasis were distant lymph nodes, followed by bone, lung, and liver.

\section{Effectiveness}

The median follow-up time was 9.1 months for patients who received CT-P6 and 32.3 months for those who received RTZ. Patients in the CT-P6 group received a median (range) of 11.1 (2-32) cycles while those in the RTZ group received a median (range) of $24.3(1-88)$ cycles. Median PFS did not differ significantly between the two treatment groups (CT-P6: 13.9 months [95\% $\mathrm{Cl}$ not available (NA)] versus RTZ: 18.4 months [95\% Cl 12.5-24.3]; $P=0.653$ ) (Fig. 2A). There was also no significant difference in median PFS between the two treatment groups regardless of HR status (HR-positive: CT-P6 10.0 months [95\% CI NA] versus RTZ 18.3 months [95\% Cl 11.5-25.1], $P=0.464 ;$ HR-negative: not reached versus 18.0 months [95\% Cl 10.3-27.3], $P=0.874$ ) (Fig. 2B and 2C). mOS was 30.0 months in patients who received treatment with CT-P6, and was not reached in the RTZ group $(P=0.880)$. There was no significant difference in mOS between the two treatment groups regardless of HR status (data not shown).

The ORR and DCR did not differ significantly between treatment groups (ORR: 78.9\% [CT-P6] versus 83.1\% [RTZ], $P=0.602$; DCR: $94.7 \%$ [CT-P6] versus $93.8 \%$ [RTZ], $P>0.999)$; there was no significant difference between the two groups in ORR or DCR according to HR status (Table 2). 
Table 2

Best overall response, based on RECIST 1.1 criteria, according to palliative treatment in patients with HER2-positive metastatic breast cancer

\begin{tabular}{|c|c|c|c|c|c|c|c|c|c|}
\hline & \multicolumn{3}{|c|}{ All patients } & \multicolumn{3}{|c|}{ HR+/HER2+ } & \multicolumn{3}{|c|}{ HR-/HER2+ } \\
\hline & $\begin{array}{l}\text { RTZ } \\
(n= \\
65)\end{array}$ & $\begin{array}{l}\text { CT-P6 } \\
(n=1 \\
38)\end{array}$ & $\begin{array}{l}\mathrm{P}- \\
\text { value }\end{array}$ & $\begin{array}{l}\text { RTZ } \\
(n= \\
29)\end{array}$ & $\begin{array}{l}\text { CT-P6 } \\
(n=16)\end{array}$ & $\begin{array}{l}\mathrm{P}- \\
\text { value }\end{array}$ & $\begin{array}{l}\text { RTZ } \\
(n= \\
36)\end{array}$ & $\begin{array}{l}\text { CT-P6 } \\
(n= \\
22)\end{array}$ & $\begin{array}{l}\mathrm{P}- \\
\text { value }\end{array}$ \\
\hline $\begin{array}{l}\text { Overall } \\
\text { response }\end{array}$ & & & $0.706^{a}$ & & & $0.902^{a}$ & & & $0.389^{a}$ \\
\hline $\mathrm{CR}, n(\%)$ & $\begin{array}{l}5 \\
(7.7)\end{array}$ & $2(5.3)$ & & $\begin{array}{l}3 \\
(10.3)\end{array}$ & $1(6.3)$ & & $\begin{array}{l}2 \\
(5.6)\end{array}$ & $\begin{array}{l}1 \\
(4.5)\end{array}$ & \\
\hline $\mathrm{PR}, n(\%)$ & $\begin{array}{l}49 \\
(75.4)\end{array}$ & $\begin{array}{l}28 \\
(73.7)\end{array}$ & & $\begin{array}{l}18 \\
(62.1)\end{array}$ & $\begin{array}{l}11 \\
(68.8)\end{array}$ & & $\begin{array}{l}31 \\
(86.1)\end{array}$ & $\begin{array}{l}17 \\
(77.3)\end{array}$ & \\
\hline $\mathrm{SD}, n(\%)$ & $\begin{array}{l}7 \\
(10.8)\end{array}$ & $\begin{array}{l}6 \\
(15.8)\end{array}$ & & $\begin{array}{l}5 \\
(17.2)\end{array}$ & $\begin{array}{l}4 \\
(25.0)\end{array}$ & & $\begin{array}{l}2 \\
(5.6)\end{array}$ & $\begin{array}{l}2 \\
(9.1)\end{array}$ & \\
\hline PD, $n(\%)$ & $\begin{array}{l}2 \\
(3.1)\end{array}$ & $2(5.3)$ & & $\begin{array}{l}2 \\
(6.9)\end{array}$ & 0 & & 0 & $\begin{array}{l}2 \\
(9.1)\end{array}$ & \\
\hline $\mathrm{NA}, n(\%)$ & $\begin{array}{l}2 \\
(3.1)\end{array}$ & 0 & & $\begin{array}{l}1 \\
(3.4)\end{array}$ & 0 & & $\begin{array}{l}1 \\
(2.8)\end{array}$ & 0 & \\
\hline $\begin{array}{l}\text { Overall } \\
\text { response } \\
\text { rate }\end{array}$ & & & 0.602 & & & $\begin{array}{l}> \\
0.999 \text { a }\end{array}$ & & & $0.409^{a}$ \\
\hline $\begin{array}{l}\text { CR/PR, } n \\
(\%)\end{array}$ & $\begin{array}{l}54 \\
(83.1)\end{array}$ & $\begin{array}{l}30 \\
(78.9)\end{array}$ & & $\begin{array}{l}21 \\
(72.4)\end{array}$ & $\begin{array}{l}12 \\
(75.0)\end{array}$ & & $\begin{array}{l}33 \\
(91.7)\end{array}$ & $\begin{array}{l}18 \\
(81.8)\end{array}$ & \\
\hline $\begin{array}{l}\text { Disease } \\
\text { control rate }\end{array}$ & & & $\begin{array}{l}> \\
0.999 \text { a }\end{array}$ & & & $0.542^{a}$ & & & $0.551^{\mathrm{a}}$ \\
\hline $\begin{array}{l}\text { CR/PR/SD, } \\
n(\%)\end{array}$ & $\begin{array}{l}61 \\
(93.8)\end{array}$ & $\begin{array}{l}36 \\
(94.7)\end{array}$ & & $\begin{array}{l}26 \\
(89.7)\end{array}$ & $\begin{array}{l}16 \\
(100.0)\end{array}$ & & $\begin{array}{l}35 \\
(97.2)\end{array}$ & $\begin{array}{l}20 \\
(90.9)\end{array}$ & \\
\hline \multicolumn{10}{|c|}{ a Fisher's exact test. } \\
\hline $\begin{array}{l}\text { CR, complete } \\
\text { not available } \\
\text { in Solid Tum }\end{array}$ & $\begin{array}{l}\text { response } \\
\text { PD, prog } \\
\text { rs versio }\end{array}$ & $\begin{array}{l}\text { HER2, h } \\
\text { essive di } \\
1.1 ; \mathrm{RT} 2\end{array}$ & $\begin{array}{l}\text { nan epid } \\
\text { ease; PR, } \\
\text { reference }\end{array}$ & $\begin{array}{l}\text { mal grc } \\
\text { artial re } \\
\text { rastuzu }\end{array}$ & $\begin{array}{l}\text { th factor } \\
\text { onse; RE } \\
\text { lab;SD, s }\end{array}$ & $\begin{array}{l}\text { ceptor 2; } \\
\text { ST 1.1, R } \\
\text { ole disea }\end{array}$ & $\begin{array}{l}\text { AR, horm } \\
\text { sponse }\end{array}$ & $\begin{array}{l}\text { ne recep } \\
\text { galuatior }\end{array}$ & $\begin{array}{l}\text { or; NA, } \\
\text { Criteria }\end{array}$ \\
\hline
\end{tabular}

Cardiac safety

Both CT-P6 and RTZ showed a manageable cardiac safety profile, assessed on the basis of LVEF (Additional file 4). Thirteen out of 38 (34.2\%) patients in the CT-P6 group experienced a decline in LVEF of $\geq 10$ percentage points from baseline, compared with $25 / 65$ (38.5\%) patients in the RTZ group ( $P=$ 0.695). Among those who received treatment with CT-P6, 2/38 (5.3\%) patients experienced a reduction in 
LVEF to $<50 \%$ at any time. One of these patients discontinued CT-P6 for 1 month before re-starting; the other continued treatment but later discontinued after 3 months owing to progressive disease. Among the patients who received treatment with RTZ, 4/65 (6.2\%) experienced a reduction in LVEF to $<50 \%$ at any time. Two of these patients discontinued treatment with RTZ, one discontinued for 1 month before restarting, and the other continued treatment with RTZ.

\section{Discussion}

To our knowledge, this is the first real-world study to show that CT-P6 has similar effectiveness and cardiac safety to RTZ in patients with HER2-positive EBC and MBC, when administered as part of dual HER2-targeted therapy with pertuzumab and docetaxel in the neoadjuvant or palliative setting.

Almost 75\% of patients with HER2-positive EBC who received CT-P6 achieved a pCR, compared with $70 \%$ of patients who received RTZ. In the EBC cohort, a significantly greater percentage of patients in the CT-P6 versus RTZ treatment group had a Ki67 index $\geq 14$ at baseline (87.5\% versus $70.5 \% ; P=0.005)$. Notably, the Ki67 index is known to be a predictive marker for pCR in patients with breast cancer who receive neoadjuvant chemotherapy. $[25,26]$ Therefore, we implemented PSM to reduce confounding bias: in the PSM cohort, there was no difference in response to treatment between the two groups, similar to the findings for the whole EBC cohort.

In the phase III randomized controlled trial that established the equivalence of CT-P6 and the reference product, no pertuzumab was added to the neoadjuvant regimen.[16] However, since the dual HER2targeted approach may further improve pCR rates compared with use of either monoclonal antibody alone,[20, 24, 27, 28] most patients with HER2-positive breast cancer who received neoadjuvant chemotherapy at our institution were treated with TCHP from 2018, when CT-P6 was approved. For this reason, we compared the efficacy of CT-P6 and RTZ among patients with HER2-positive EBC who received TCHP in the neoadjuvant setting. The current results confirm for the first time in routine clinical practice that CT-P6 is at least as effective as RTZ when administered as part of dual HER2-targeted therapy with pertuzumab.

Our study also provides the first real-world evidence that CT-P6 is as effective as RTZ in patients with HER2-positive MBC, when administered with pertuzumab and docetaxel. In the CLEOPATRA trial, first-line treatment with pertuzumab-trastuzumab-docetaxel improved PFS in patients with HER2-positive MBC compared with placebo-trastuzumab-docetaxel (18.5 versus 12.4 months; $P<0.001)$,[24] and extended mOS (56.5 versus 40.8 months; $P<0.001$ ). [22] A real-world study of patients with HER2-positive advanced breast cancer in Italy observed a response rate of $77.3 \%$ with pertuzumab-trastuzumabtaxane, along with a median PFS of 21 months.[29] These results are consistent with those of the current study, in which the ORR was $78.9 \%$ with CT-P6-pertuzumab-docetaxel and $83.1 \%$ with RTZpertuzumab-docetaxel. There was also no significant difference in median PFS between patients who received CT-P6 and those who received RTZ in the current study (13.9 versus 18.4 months; $P=0.653$ ). While not statistically significant, the numerical difference in PFS between the treatment groups may 
reflect the shorter follow-up time for the CT-P6 group than RTZ (9.1 versus 32.3 months, respectively). However, since a relatively large number of censored events occurred early in the follow-up period for CTP6, but later in the follow-up period for RTZ, a longer-term follow-up analysis will be required to confirm the equivalence of CT-P6 and RTZ with respect to median PFS.

Use of trastuzumab has historically been associated with an increased risk of cardiotoxicity,[6] most often in the form of an asymptomatic decline in LVEF. In the current study, neither CT-P6 nor RTZ showed serious cardiac toxicity profiles. No patients with EBC experienced a reduction in LVEF of $<50 \%$ in either treatment group, consistent with the low cardiotoxicity observed in patients with EBC treated with RTZ or CT-P6 in the neoadjuvant[16, 21] and adjuvant[17, 21] settings. In the current study, $2 / 38(5.3 \%)$ patients with MBC experienced a decline in LVEF to $<50 \%$ with CT-P6, as did 4/65 (6.2\%) patients in the RTZ group. A similar decline in LVEF was observed in $6.1 \%$ of patients treated with pertuzumabtrastuzumab-docetaxel in the CLEOPATRA trial.[22]

One strength of our study is that it included a broader patient population, that is more representative of the patient population as a whole, than the highly selected individuals recruited into clinical trials. This is also the first study to compare the efficacy and safety of CT-P6 and RTZ in both EBC and MBC based on data from two cancer centers in Korea. There were also limitations: firstly, the study had a retrospective design and a relatively small sample size. Therefore, further prospective and larger studies are warranted. Secondly, the follow-up time for patients who received CT-P6 was shorter than for those who received RTZ. While the analysis found no significant difference in median PFS with CT-P6 versus RTZ, a longer follow-up period will be required to confirm their equivalence with respect to PFS, as well as OS.

Despite the established safety and efficacy profile of RTZ, the high cost of the drug remains a barrier to access, particularly in healthcare systems with fewer resources.

For example, a study in China found that patients with HER2-positive EBC who lived in areas with a relatively high gross domestic product were more likely to receive RTZ than those in areas with a lower gross domestic product.

[30] The same study reported better survival outcomes in patients with EBC or MBC who received treatment with RTZ, illustrating how the high cost of RTZ can deprive patients of effective treatments. Biosimilars can provide treatment that is as safe and effective as RTZ, but at a significantly reduced cost. [8]

\section{Conclusion}

This real-world study suggests that the biosimilar CT-P6 has similar efficacy and cardiac safety to RTZ in HER2-positive EBC and MBC, when administered as part of dual HER2-targetd therapy with pertuzumab and chemotherapy in the neoadjuvant or palliative first-line setting. CT-P6 was well tolerated, with a cardiac safety profile similar to that of the reference product. The increased use of biosimilars such as CT-P6 has the potential to boost access to life-extending treatments for women with HER2-positive breast cancer. 


\section{Declarations}

\section{Ethics approval and consent to participate}

This study was reviewed and approved by the institutional review boards (IRBs) of Yonsei Cancer Center (IRB no. 4-2014-0551) and Gangnam Severance Hospital (IRB no. 3-2020-0090). Informed consent was waived due to the retrospective nature of the study, which was conducted in line with Good Clinical Practice guidelines and the Declaration of Helsinki.

\section{Consent for publication}

Not applicable.

\section{Availability of data and materials}

Data will be available on request.

\section{Competing interests}

The authors have declared no conflicts of interest.

\section{Funding information}

The authors received no specific funding for this work.

\section{Author's Contributions}

S.J.B.: Formal analysis, Investigation, Resources, Data curation, Writing - original draft, Visualization. J.H.K.: Conceptualization, Methodology, Formal analysis, Investigation, Resources, Writing- original draft. S.G.A.: Conceptualization, Methodology, Investigation, Resources. H.C.J.: Investigation, Resources. J.S.: Investigation, Resources. G.M.K.: Investigation, Resources. M.H.K.: Investigation, Resources. S.I.K.: Investigation, Resources. S.P.: Investigation, Resources, H.S.P.: Investigation, Resources, J.Y.K.: Investigation, Resources, J.J.: Conceptualization, Methodology, Investigation, Resources, Writing - original draft, Writing - review \& editing, Supervision, Project administration.

All authors read and approved the final manuscript.

\section{Acknowledgments}


Medical writing support (including development of a first draft, based on an outline provided by the authors, and subsequent drafts in consultation with the authors, assembling tables and figures, collating author comments, copyediting, fact checking, and referencing) was provided by Beatrice Tyrrell, DPhil, at Aspire Scientific Limited (Bollington, UK), and funded by Celltrion Healthcare (Incheon, Republic of Korea).

\section{References}

1. Slamon DJ, Godolphin W, Jones LA, Holt JA, Wong SG, Keith DE, Levin WJ, Stuart SG, Udove J, Ullrich $A$, et al. Studies of the HER-2/neu proto-oncogene in human breast and ovarian cancer. Science. 1989;244(4905):707-12.

2. Buzdar AU, Ibrahim NK, Francis D, Booser DJ, Thomas ES, Theriault RL, Pusztai L, Green MC, Arun BK, Giordano SH, et al. Significantly higher pathologic complete remission rate after neoadjuvant therapy with trastuzumab, paclitaxel, and epirubicin chemotherapy: results of a randomized trial in human epidermal growth factor receptor 2-positive operable breast cancer. J Clin Oncol. 2005;23(16):3676-85.

3. Untch M, Rezai M, Loibl S, Fasching PA, Huober J, Tesch H, Bauerfeind I, Hilfrich J, Eidtmann H, Gerber B, et al. Neoadjuvant treatment with trastuzumab in HER2-positive breast cancer: results from the GeparQuattro study. J Clin Oncol. 2010;28(12):2024-31.

4. Gianni L, Eiermann W, Semiglazov V, Manikhas A, Lluch A, Tjulandin S, Zambetti M, Vazquez F, Byakhow $M$, Lichinitser $M$, et al. Neoadjuvant chemotherapy with trastuzumab followed by adjuvant trastuzumab versus neoadjuvant chemotherapy alone, in patients with HER2-positive locally advanced breast cancer (the NOAH trial): a randomised controlled superiority trial with a parallel HER2-negative cohort. Lancet. 2010;375(9712):377-84.

5. Cameron D, Piccart-Gebhart MJ, Gelber RD, Procter M, Goldhirsch A, de Azambuja E, Castro G Jr, Untch M, Smith I, Gianni L, et al. 11 years' follow-up of trastuzumab after adjuvant chemotherapy in HER2-positive early breast cancer: final analysis of the HERceptin Adjuvant (HERA) trial. Lancet. 2017;389(10075):1195-205.

6. Slamon DJ, Leyland-Jones B, Shak S, Fuchs H, Paton V, Bajamonde A, Fleming T, Eiermann W, Wolter $\mathrm{J}$, Pegram $\mathrm{M}$, et al. Use of chemotherapy plus a monoclonal antibody against HER2 for metastatic breast cancer that overexpresses HER2. N Engl J Med. 2001;344(11):783-92.

7. Balduzzi S, Mantarro S, Guarneri V, Tagliabue L, Pistotti V, Moja L, D'Amico R. Trastuzumabcontaining regimens for metastatic breast cancer. Cochrane Database Syst Rev 2014(6):CD006242.

8. Lammers P, Criscitiello C, Curigliano G, Jacobs I. Barriers to the use of trastuzumab for HER2 + breast cancer and the potential impact of biosimilars: A physician survey in the United States and emerging markets. Pharmaceuticals (Basel). 2014;7(9):943-53.

9. Jeong SA, Choi JM, Park JM, Lee JY, Lee SJ, Lee SY, Lee SY, Park YA, Jeong HJ, Song YC, et al. Mechanism of action of the trastuzumab biosimilar CT-P6. Expert Opin Biol Ther. 2019;19(10):108595. 
10. Fu C, Stebbing J, Esteva FJ. Clinical development of CT-P6 in HER2 positive breast cancer. Expert Opin Biol Ther. 2019;19(10):987-92.

11. Lamb YN. SB3 (Ontruzant $\left.{ }^{(\mathrm{R})}\right)$ : A trastuzumab biosimilar. BioDrugs. 2018;32(3):293-6.

12. Dhillon S. ABP 980: A trastuzumab biosimilar. BioDrugs. 2018;32(5):511-4.

13. Paik J. PF-05280014: A trastuzumab biosimilar. BioDrugs. 2018;32(5):515-8.

14. Herzuma. prescribing information https://www.accessdata.fda.gov/drugsatfda_docs/label/2018/761091s000lbl.pdf.

15. Herzuma summary of product characteristics [https://.

16. Stebbing J, Baranau Y, Baryash V, Manikhas A, Moiseyenko V, Dzagnidze G, Zhavrid E, Boliukh D, Stroyakovskii D, Pikiel J, et al. CT-P6 compared with reference trastuzumab for HER2-positive breast cancer: a randomised, double-blind, active-controlled, phase 3 equivalence trial. Lancet Oncol. 2017;18(7):917-28.

17. Esteva FJ, Baranau YV, Baryash V, Manikhas A, Moiseyenko V, Dzagnidze G, Zhavrid E, Boliukh D, Stroyakovskiy D, Pikiel J, et al. Efficacy and safety of CT-P6 versus reference trastuzumab in HER2positive early breast cancer: updated results of a randomised phase 3 trial. Cancer Chemother Pharmacol. 2019;84(4):839-47.

18. Stebbing J, Baranau Y, E Z VBAMVMGD, D B, D S, J P et al: 3-year follow-up of a phase III trial comparing the efficacy and safety of neoadjuvant and adjuvant trastuzumab and its biosimilar CTP6 in HER2 positive early breast cancer (EBC). Ann Oncol 2019, 30 (supplement 5):v63-v64.

19. Franklin MC, Carey KD, Vajdos FF, Leahy DJ, de Vos AM, Sliwkowski MX. Insights into ErbB signaling from the structure of the ErbB2-pertuzumab complex. Cancer Cell. 2004;5(4):317-28.

20. Gianni L, Pienkowski T, Im YH, Roman L, Tseng LM, Liu MC, Lluch A, Staroslawska E, de la HabaRodriguez J, Im SA, et al. Efficacy and safety of neoadjuvant pertuzumab and trastuzumab in women with locally advanced, inflammatory, or early HER2-positive breast cancer (NeoSphere): a randomised multicentre, open-label, phase 2 trial. Lancet Oncol. 2012;13(1):25-32.

21. Gianni L, Pienkowski T, Im YH, Tseng LM, Liu MC, Lluch A, Staroslawska E, de la Haba-Rodriguez J, Im SA, Pedrini JL, et al. 5-year analysis of neoadjuvant pertuzumab and trastuzumab in patients with locally advanced, inflammatory, or early-stage HER2-positive breast cancer (NeoSphere): a multicentre, open-label, phase 2 randomised trial. Lancet Oncol. 2016;17(6):791-800.

22. Swain SM, Baselga J, Kim SB, Ro J, Semiglazov V, Campone M, Ciruelos E, Ferrero JM, Schneeweiss $A$, Heeson $S$, et al. Pertuzumab, trastuzumab, and docetaxel in HER2-positive metastatic breast cancer. N Engl J Med. 2015;372(8):724-34.

23. Schneeweiss A, Chia S, Hickish T, Harvey V, Eniu A, Hegg R, Tausch C, Seo JH, Tsai YF, Ratnayake J, et al. Pertuzumab plus trastuzumab in combination with standard neoadjuvant anthracyclinecontaining and anthracycline-free chemotherapy regimens in patients with HER2-positive early breast cancer: a randomized phase II cardiac safety study (TRYPHAENA). Ann Oncol. 2013;24(9):2278-84. 
24. Baselga J, Cortes J, Kim SB, Im SA, Hegg R, Im YH, Roman L, Pedrini JL, Pienkowski T, Knott A, et al. Pertuzumab plus trastuzumab plus docetaxel for metastatic breast cancer. N Engl J Med. 2012;366(2):109-19.

25. Kurozumi S, Inoue K, Takei H, Matsumoto H, Kurosumi M, Horiguchi J, Takeyoshi I, Oyama T. ER, PgR, Ki67, p27(Kip1), and histological grade as predictors of pathological complete response in patients with HER2-positive breast cancer receiving neoadjuvant chemotherapy using taxanes followed by fluorouracil, epirubicin, and cyclophosphamide concomitant with trastuzumab. BMC Cancer. 2015;15:622.

26. Petit T, Wilt M, Velten M, Millon R, Rodier JF, Borel C, Mors R, Haegelé P, Eber M, Ghnassia JP. Comparative value of tumour grade, hormonal receptors, Ki-67, HER-2 and topoisomerase II alpha status as predictive markers in breast cancer patients treated with neoadjuvant anthracycline-based chemotherapy. European journal of cancer (Oxford England: 1990). 2004;40(2):205-11.

27. Diaz-Redondo T, Lavado-Valenzuela R, Jimenez B, Pascual T, Galvez F, Falcon A, Alamo MDC, Morales $\mathrm{C}$, Amerigo $\mathrm{M}$, Pascual $\mathrm{J}$, et al. Different pathological complete response rates according to PAM50 subtype in HER2 + breast cancer patients treated with neoadjuvant pertuzumab/trastuzumab vs. trastuzumab plus standard chemotherapy: An analysis of real-world data. Front Oncol. 2019;9:1178.

28. Fasching PA, Hartkopf AD, Gass P, Haberle L, Akpolat-Basci L, Hein A, Volz B, Taran FA, Nabieva N, Pott $B$, et al. Efficacy of neoadjuvant pertuzumab in addition to chemotherapy and trastuzumab in routine clinical treatment of patients with primary breast cancer: a multicentric analysis. Breast Cancer Res Treat. 2019;173(2):319-28.

29. Gamucci T, Pizzuti L, Natoli C, Mentuccia L, Sperduti I, Barba M, Sergi D, lezzi L, Maugeri-Sacca M, Vaccaro A, et al. A multicenter REtrospective observational study of first-line treatment with PERtuzumab, trastuzumab and taxanes for advanced HER2 positive breast cancer patients. RePer Study. Cancer Biol Ther. 2019;20(2):192-200.

30. Li J, Wang S, Wang Y, Wang X, Wang H, Feng J, Zhang Q, Sun T, Ouyang Q, Yin Y, et al. Disparities of trastuzumab use in resource-limited or resource-abundant regions and its survival benefit on HER2 positive breast cancer: A real-world study from China. Oncologist. 2017;22(11):1333-8.

\section{Figures}




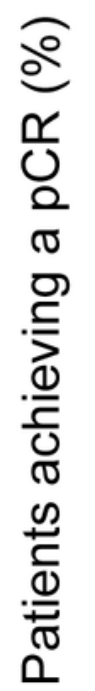

100
80
60
40
20

RTZ

CT-P6

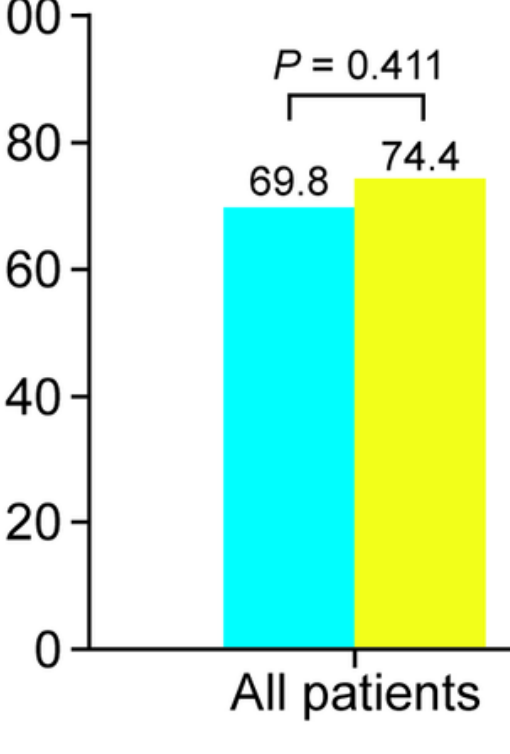

(a)

$P=0.943$



$83.3 \quad 83.8$

$P=0.206$

57.8

44.4

44.4 


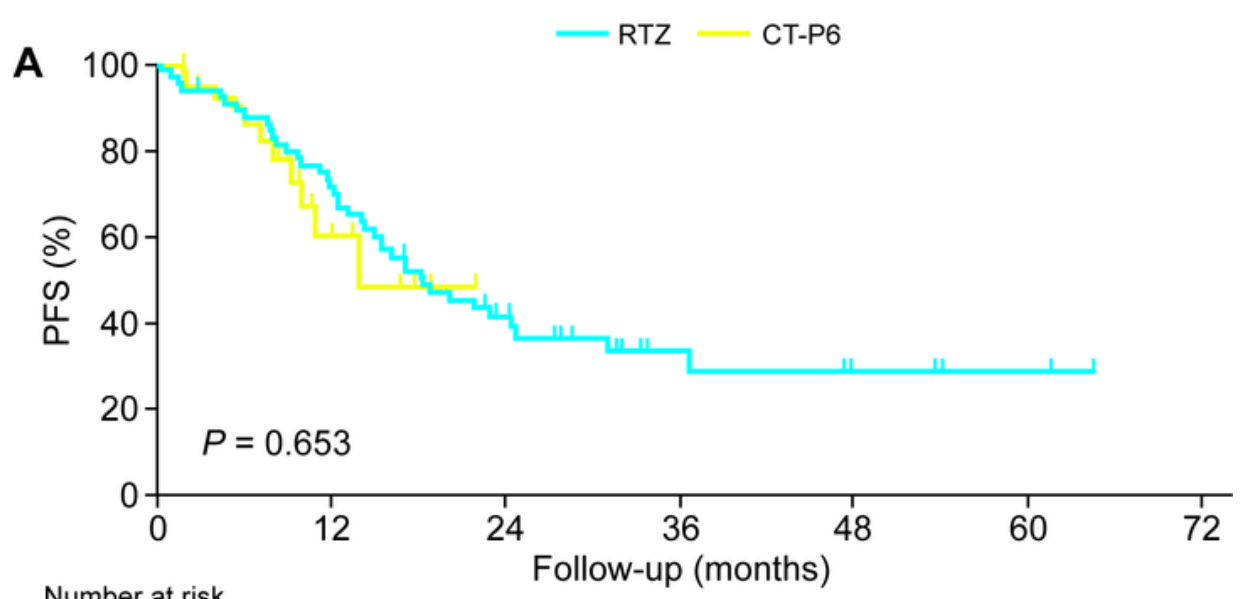

$\begin{array}{lcccccc}\text { Number at risk } & & & & & \\ \text { RTZ } & 65 & 44 & 18 & 7 & 4 & 0 \\ \text { CT-P6 } & 38 & 9 & 0 & 0 & 0 & 0\end{array}$
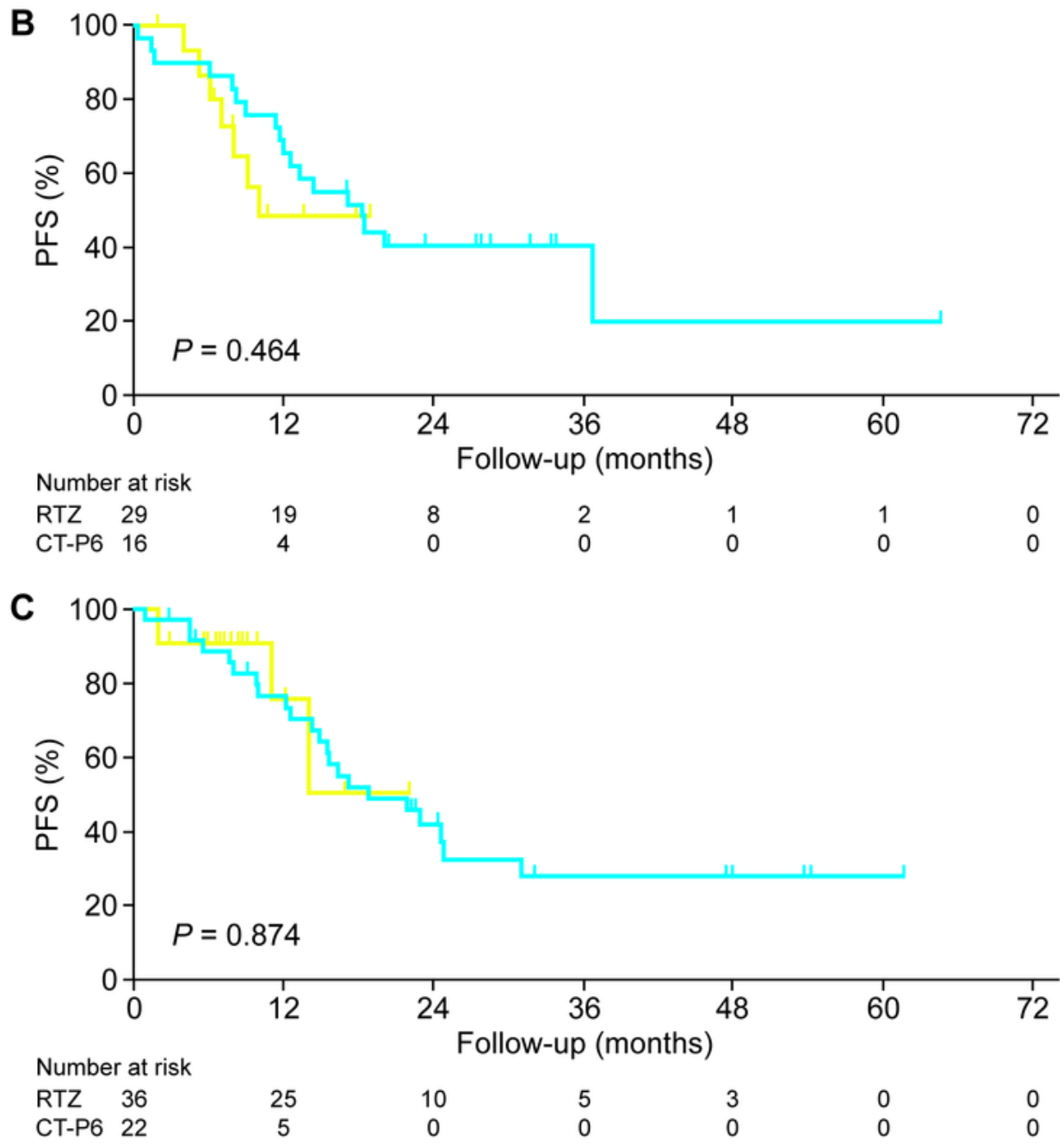

Figure 2

PFS for HER2-positive metastatic breast cancer patients who received palliative treatment with CT-P6 or RTZ for (A) all patients, (B) HR+/HER2+ patients, and (C) HR-/HER2+ patients. HER2, human epidermal growth factor receptor 2; HR, hormone receptor; PFS, progression-free survival; RTZ, reference trastuzumab. 


\section{Supplementary Files}

This is a list of supplementary files associated with this preprint. Click to download.

- CTP6realworldmanuscriptadditionalfile1.docx

- CTP6realworldmanuscriptadditionalfile2.docx

- CTP6realworldmanuscriptadditionalfile3.docx

- CTP6realworldmanuscriptadditionalfile4.docx 